\title{
Turbulence in Molecular Clouds
}

\author{
Enrique Vázquez-Semadeni \\ Instituto de Astronomía, UNAM \\ Apartado Postal 70-264, México D.F. MEXICO
}

\begin{abstract}
In this course we review the theory of incompressible homogeneous turbulence at an elementary level, and discuss the similarities and differences expected in the compressible case, relevant to the interstellar medium and molecular clouds. We stress that a general definition of turbulence applicable to the compressible case should not rely on the Kolmogorov $k^{-5 / 3}$ spectrum nor on an energy cascade from large to small scales. Instead, we discuss the various possibilities for the energy spectrum of compressible turbulence, which numerical simulations suggest should be $\sim k^{-2}$, and the nature of the cascades, if at all present. We then discuss issues concerning molecular clouds which are likely to be directly related to turbulence, such as cloud formation, cloud structure, and cloud support against gravity.
\end{abstract}

To appear in "Millimetric and Sub-Millimetric Astronomy. INAOE 1996 Summer School".

\section{Introduction}

In recent years, a wide variety of scientific disciplines have come to the realization that nonlinear phenomena are the norm rather than the exception. Most physical, astronomical, biological, social and economic systems are strongly nonlinear, and in fact the time is ripe for a change in our categorization of such systems. While our current classifications of dynamical behavior are based on a "linear vs. nonlinear" scheme, intrinsically attributing preponderance to linear systems, a "complex vs. non-complex" classification would probably be more appropriate, reflecting the fact that most systems in Nature and society are complex.

Turbulent flows are a prime example of complex systems, and it is well known that a complete theory even of incompressible turbulence does not exist. By this it is meant that some specific statistical properties of turbulent flows, such as the energy spectrum and the higher order correlation functions, cannot be derived directly from the equations of motion without the introduction of simplifying assumptions. Nevertheless, phenomenolog- 
ical theories exist for both three-dimensional (Kolmogorov 1941) and twodimensional (Kraichnan 1967) turbulence in incompressible flows, which have been extremely influential. In fact, the Kolmogorov theory has almost become synonymous with turbulence. However, it will be argued in this course that such an identification is likely not to be adequate for compressible turbulence, and thus a more appropriate definition of turbulence is that a) it contains an extremely large number of excited degrees of freedom (or modes); $b$ ) the modes are able to nonlinearly exchange excitation; c) the system is unpredictable in the sense of exhibiting sensitivity to initial conditions, and d) the system is mixing (see, e.g., Scalo 1987; Lesieur 1990; Frisch 1995). Property c) is a distinctive feature of chaotic systems.

The interstellar medium (ISM) in particular is an extremely complex system, including gaseous, dust, cosmic ray and magnetic field components in a strongly turbulent state. As discussed in the Virial Theorem chapter of this book, the gaseous and magnetic components of the ISM are probably well described by the magnetohydrodynamic (MHD) equations (see, e..g., Cowling 1976; Spitzer 1978; Shore 1992; Shu 1992). In this course, we will discuss some basic theoretical aspects of compressible turbulence, remarking its differences with the incompressible case $(\S 2$.), and then review some observational and theoretical work regarding several aspects of turbulence and molecular clouds, namely cloud formation, cloud structure and cloud support and star formation (§ 3.), emphasizing on recent developments. Extensive reviews of earlier work can be found in Dickman (1985) and Scalo (1987). Finally, $§ 4$ presents a summary and conclusions.

\section{Basic Theory}

\subsection{Incompressible hydrodynamic turbulence}

In this section we discuss the basic notions necessary for the subsequent discussions. Comprehensive presentations of homogeneous turbulence can be found in Batchelor (1953), Tennekes \& Lumley (1972), Rose \& Sulem (1978) and Lesieur (1990).

The Navier-Stokes equation. The full MHD equations have already been presented in the chapter on the Virial Theorem. Here we just discuss the incompressible momentum conservation equation without the Lorentz, Coriolis, or other external forces. When including the constitutive relation for stress in a newtonian fluid (see, e.g., Currie 1974) the momentum equation is referred to as the Navier-Stokes (NS) equation. It reads:

$$
\frac{\partial \mathbf{u}}{\partial t}+\mathbf{u} \cdot \nabla \mathbf{u}=-\frac{\nabla P}{\rho}+\nu \nabla^{2} \mathbf{u}
$$


where $\mathbf{u}$ is the velocity field, $\rho$ is the density, $P$ is the pressure, and $\nu$ is the kinematic viscosity. Additionally, the incompressibility condition $d \rho / d t=0$ applied to the continuity (or mass conservation) equation yields

$$
\nabla \cdot \mathbf{u}=0
$$

As mentioned in the Virial Theorem chapter, the second term in the LHS of eq. (11) is called the advective or nonlinear term, and is the one responsible for turbulent transport of momentum (the $j$-th component of the velocity transporting the $i$-th component of the momentum). On the other hand, viscosity tends to unify the motion of fluid parcels. Thus, a measure of the "amount of turbulence" present in the flow is given by the ratio of the advective to the viscous term, known as the Reynolds number Re. Dimensionally,

$$
R e \equiv \frac{|\mathbf{u} \cdot \nabla \mathbf{u}|}{\left|\nu \nabla^{2} \mathbf{u}\right|} \sim \frac{U L}{\nu},
$$

where $U$ is a characteristic value of the velocity, and $L$ is the characteristic length scale of the flow.

Fourier decomposition. Much of turbulence theory is done in Fourier space, as it permits a direct treatment of length scales - the wavelengths associated to the Fourier modes. We take a Fourier decomposition of the velocity field of the form $\mathbf{u}(\mathbf{x}, t)=\int u_{\mathbf{k}}(t) e^{i \mathbf{k} \cdot \mathbf{x}} d^{3} k$, where $\mathbf{k}$ is the vector Fourier mode, with associated wavenumber $k=2 \pi / \lambda, \lambda$ is the mode wavelength, and $\mathbf{u}_{\mathbf{k}}$ is the corresponding mode amplitude. Under the assumption of periodic boundary conditions in a domain of size $L$, the wavevectors $\mathbf{k}$ have discrete components, multiples of $2 \pi / L$. Choosing a box length $L=2 \pi$ thus gives wavevectors with integer components. In Fourier space, the NS equation becomes

$$
\frac{d \mathbf{u}_{\mathbf{k}}}{d t}+i \sum_{\mathbf{p}+\mathbf{q}=\mathbf{k}}\left[\left(\mathbf{k} \cdot \mathbf{u}_{\mathbf{p}}\right) \mathbf{u}_{\mathbf{q}}-\frac{\mathbf{k}}{k^{2}}\left(\mathbf{k} \cdot \mathbf{u}_{\mathbf{p}}\right)\left(\mathbf{k} \cdot \mathbf{u}_{\mathbf{q}}\right)\right]=-\nu k^{2} \mathbf{u}_{\mathbf{k}}
$$

with the incompressibility condition

$$
\mathbf{k} \cdot \mathbf{u}_{\mathbf{k}}=0 \text {. }
$$

Several points are worth noting. The first term in the brackets is the advective term. The second term in the brackets is the pressure gradient term. Interestingly, in the incompressible case, the pressure gradient term can be solved for in terms of the velocity field in Fourier space. This is because the incompressible equations behave as if the speed of sound were infinite, short-circuiting the longer interaction path for the compressible case, in which compressive motions cause density fluctuations via the $\nabla \cdot \mathbf{u}$ term in 
the continuity equation, which in turn cause pressure fluctuations (mediated by the speed of sound), which finally feed back in the momentum equation via the $\nabla P$ term.

Secondly, both the advective and the pressure terms are seen to be nonlinear, i.e., to involve products of pairs of velocity modes. Moreover, it is seen that all modes $\mathbf{p}$ and $\mathbf{q}$ such that $\mathbf{p}+\mathbf{q}=\mathbf{k}$ enter the RHS of the equation for mode $\mathbf{k}$, so that energy (or, more generally, excitation) is exchanged among all Fourier modes. This process is known as nonlinear transfer. The term excitation is used to include transfer among modes of different kinds (density, velocity, internal energy, etc.), which will be appropriate for the compressible case.

Third, note that eq. (1) actually represents an enormously large number of coupled ordinary differential equations (ODEs) for the Fourier amplitudes $\mathbf{u}_{\mathbf{k}}$. For example, if we were to perform a numerical simulation with 1000 grid points per dimension in three dimensions, this would amount to roughly $10^{9}$ Fourier modes, or degrees of freedom. Although very large for current computer capabilities, this is still a very small resolution for real problems. It was pointed out in the Virial Theorem chapter that there is at least a factor of $10^{5}$ between the molecular mean free path and the characteristic scales of the system in most ISM structures, implying at least $10^{15}$ degrees of freedom (or equations, in the Fourier description). Thus, the Fourier description trades the complexity of a single partial differential equation for a very large number of ODEs.

Fourth, although not readily seen from the equations, turbulent flows generally exhibit sensitivity to initial conditions, i.e., infinitesimal changes in the initial conditions can lead to markedly different states after finite times (see, e.g., Ott 1993).

Finally, note that the incompressibility condition clearly shows that no longitudinal modes exist in incompressible flow.

Energy spectrum and correlation function. The Fourier description leads naturally to the definition of an important quantity in turbulence theory, namely the energy spectrum $E(k)$. This is defined as the kinetic energy (per unit mass) contained in modes with wavenumbers in the interval $(k-$ $1 / 2, k+1 / 2]$. This amounts to summing up the energies $\left|\mathbf{u}_{\mathbf{k}}\right|^{2}$ of all modes in a spherical shell of thickness $\Delta k=1$ and of radius $k$. Mathematically,

$$
\begin{aligned}
E \equiv & \frac{1}{2} \int|\mathbf{u}(\mathbf{x})|^{2} d^{3} x \\
& =\frac{1}{2} \int\left|\mathbf{u}_{\mathbf{k}}\right|^{2} d^{3} k \\
& =\frac{1}{2} \int_{0}^{\infty} E(k) d k
\end{aligned}
$$


where the second equality follows from the well-known result for complex variables, Parseval's relation. Thus

$$
E(k)=\frac{1}{2} \iint\left|\mathbf{u}_{\mathbf{k}}\right|^{2} k^{2} \sin \theta_{k} d \theta_{k} d \phi_{k}
$$

The exponent of $k$ in the spectrum is commonly referred to as the spectral index or slope, the latter referring to the slope of the spectrum in a log-log plot.

It can be readily shown that the energy spectrum is actually the Fourier transform of the so-called auto-correlation function. This is defined as $C(\mathbf{r}) \equiv\langle\mathbf{u}(\mathbf{x}) \cdot \mathbf{u}(\mathbf{x}+\mathbf{r})\rangle / \sigma^{2}$, where $\sigma$ is the velocity variance. This function measures the probability that two points separated by a distance $\mathbf{r}$ have the same velocity. A characteristic length is the correlation length, which is the $e$-folding distance for the correlation function. Note that the correlation function can also be defined for the density field, as in Cosmology, or for products of different variables, in which case it is called a "cross correlation". A related quantity, the structure function, is defined as $S(\mathbf{r}) \equiv\left\langle|\mathbf{u}(\mathbf{x})-\mathbf{u}(\mathbf{x}+\mathbf{r})|^{2}\right\rangle / \sigma^{2}$.

Kolmogorov-Obukhov theory. In 1941, Kolmogorov and Obukhov independently derived the expected scalings of all powers of the structure functions as functions of separation $r$ for homogeneous turbulence (i.e., whose statistical properties are independent of position). From there, the form of the energy spectrum readiliy follows. This result is a milestone in turbulence theory, and is often referred to as the K41 theory. It is based on the following assumptions:

i) The Reynolds number is large enough that a very large range of scales are active in the flow.

ii) Energy is injected primarily at large scales and dissipated at small scales (as can be seen from the $k^{2}$ dependence of the viscous term in eq. 畉).

iii) Energy transfer is local in Fourier space, i.e., it occurs mainly among similar wavenumbers. Together with the two previous assumptions, this implies that there is an intermediate range of wavenumbers in which energy can only cascade from large to small scales (small to large $k$ ). This is called the inertial range of wavenumbers.

iv) The system is in statistical equilibrium, so that the rates of energy injection at large scales, of transfer at intermediate scales and dissipation at small scales are equal. In consequence, the transfer rate in the inertial range is independent of wavenumber $k$.

With these assumptions, the form of the spectrum in the inertial range can be estimated from dimensional analysis as follows. The energy transfer rate for eddies (vortices) of scale size $l$ can be written as $\epsilon \sim V_{l}^{2} / \tau_{l}$, where $v_{l}$ is the characteristic velocity difference accross the eddy, and $\tau_{l}=l / v_{l}$ is the 
characteristic or eddy turnover time at scale $l$. Thus, the velocity differences scale with separation $l$ as

$$
v_{l}=(\epsilon l)^{1 / 3} \text {. }
$$

Now, the characteristic velocity difference at scale $l$ is related to the energy spectrum by

$$
v_{l}^{2}=2 \int_{2 \pi / l}^{\infty} E(k) d k
$$

Assuming that the spectrum has a power-law dependence on wavenumber $k^{-n}$, and substituting the scaling relation (8), we obtain $l^{2 / 3} \sim \int_{2 \pi / l}^{\infty} k^{-n} d k$, implying $n=5 / 3$. This is the celebrated Kolmogorov " $-5 / 3$ law", and has been verified experimentally (e.g., Grant et al. 1962). A more detailed discussion of this phenomenological derivation can be found in Rose \& Sulem (1978).

In summary, the K41 theory brings in two fundamental concepts, which sometimes are even considered as the trademarks of turbulent flows: a $k^{-5 / 3}$ energy spectrum and the concept of an energy cascade from large to small scales. We will see in $\$ 2.2$., however, that compressible turbulence is not necessarily characterized by these properties. Furthermore, it should also be stressed that even for incompressible flows the K41 theory does not always hold. For example, for sheared flows, Malerud et al. (1995) have found that the structure function exhibits power laws different from that expected in the K41 theory. Thus, K41 theory is only expected to apply when the assumptions of incompressibility, homogeneity, forcing at large scales, large Reynolds number (large range of excited scales) and statistical equilibrium are satisfied.

Finally, it should be remarked that the energy spectrum loses a considerable amount of information due to the averaging over angles in $k$-space. Armi \& Flament (1985) have shown that the truly relevant information concerning the structure of turbulent flows resides not in the energy spectrum, but in the complex phases of the Fourier modes, which are lost upon angle-averaging. They accomplished this by considering an ocean image, taking its Fourier transform, performing alterations on the Fourier modes, and then inverse-transforming the image. When the alterations amounted to changing the spectral slope of the field, only minor diferences in contrast were obtained. However, when the alterations amounted to scrambling the phases of the modes in a random manner, all the structural information of the image was lost. Thus, the energy spectrum is by no means completely determinant of the type of flow present in the fluid.

Two-dimensional turbulence. Two-dimensional (2D) turbulence has received comparable attention to the three-dimensional (3D) case among the turbulence community for a number of reasons. First, the large-scale motions of the Earth's oceans and athmosphere are very nearly 2D. Second, resolutions 
large enough to obtain fully developed turbulence in numerical simulations had only been achieved in 2D until very recently. Finally, 2D turbulence exhibits distinctive physical properties which constitute a challenging theoretical problem on its own right. Exhaustive discussions on 2D turbulence can be found, for example, in Kraichnan \& Montgomery (1980) and Lesieur (1990).

For 2D turbulence, the shape of the energy spectrum was studied by Kraichnan (1967), on the basis of a dimensional argument similar to Kolmogorov's for the cascade of enstrophy, the mean squared vorticity of the flow. The vorticity is defined as $\omega \equiv \nabla \times \mathbf{u}$ and the enstrophy as $\Omega \equiv$ $(1 / 2) \int \omega^{2} d V$. Assuming that energy is injected into the system at wavenumbers $k_{f}$ and that wavenumbers both larger and smaller than $k_{f}$ are present, Kraichnan predicted that in fact two different spectral slopes should be found in 2D: a -3 slope at wavenumbers larger than $k_{f}$ (scales smaller than $2 \pi / k_{f}$ ), and a $-5 / 3$ slope at wavenumbers smaller than $k_{f}$ (scales larger than $2 \pi / k_{f}$ ). Furthermore, he predicted that a direct (from larger to smaller scales) cascade of enstrophy should occur in the $k^{-3}$ range, while an inverse (from smaller to larger scales) cascade of energy should be present in the $k^{-5 / 3}$ range. These predictions have been verified numerically (Herring et al 1974; Frisch \& Sulem 1986), and constitute the standard reference against which to compare numerical results in the $2 \mathrm{D}$ compressible case, equivalent to the Kolmogorov spectrum in the 3D case.

It should nevertheless be pointed out that the nature of the enstrophy cascade and the slope of the energy spectrum for 2D flows are not completely settled. Numerical simulations (e.g., Brachet et al 1988) have suggested that in decaying (i.e., non-forced) flows, distinct evolutionary phases arise. Before small enough scales are excited, the flow develops "vorticity shocks", with a corresponding $k^{-4}$ spectrum analogous to that of a field of velocity discontinuities (\$2.2.). At later stages, once the dissipative scales have been excited, there appears to be a transition to a $k^{-3}$ spectrum. Moreover, in 2D incompressible turbulence, there is a strong tendency towards the formation of long-lived, large-scale "coherent" vortices which survive much longer after the small-scale turbulence has been dissipated by viscosity. The merging of such vortices can alter the slope of the energy spectrum (see Lesieur 1990, sect. IX.3.2 and references therein). In any case, for forced 2D turbulence, the $k^{-3}$ spectrum is the expected equilibrium spectrum, and in what follows, we will refer to the Kolmogorov and Kraichnan spectra as the statistical equilibrium spectra for $3 \mathrm{D}$ and $2 \mathrm{D}$ respectively.

\subsection{Compressible turbulence}

The compressible case is clearly much more complex than the incompressible one. The simplest case of a polytropic gas in which the pressure and the density are related by $P \sim \rho^{\gamma}$, where $\gamma$ is the polytropic exponent, in- 
volves already two coupled, partial differential equations (PDEs) (continuity and momentum). Full thermodynamics and magnetic fields add one further PDE each. Self-gravity adds the Poisson equation (see the Virial Theorem chapter in this book). Thus, self-gravitating, MHD, fully thermodynamic compressible turbulence may have little to do with the incompressible case. In the present section we discuss the similarities as well as the differences.

Density fluctuations in isothermal flow. A dimensional analysis similar to the one used in the definition of the Reynolds number in the incompressible case can be used now to estimate the order of magnitude of the density fluctuations in isothermal flow, for which $\nabla P \propto c^{2} \nabla \rho$, with $c$ the isothermal sound speed. If the magnitudes of the pressure and the advective terms are comparable, we find $|\nabla P| \sim \rho|\mathbf{u} \cdot \nabla \mathbf{u}| \Rightarrow c^{2}|\nabla \rho| \sim \rho U^{2} / L$, and thus

$$
\frac{\delta \rho}{\rho} \sim M_{a}^{2}
$$

where $M_{a} \equiv U / c$ is the Mach number corresponding to the characteristic turbulent velocity $U$. Note that we have approximated $\nabla \rho$ by $\delta \rho / L$ and $|\nabla \mathbf{u}|$ by $U / L$, since the mean velocity of the flow is assumed to be zero. Note also the resemblance of relation (10) with the density jump associated with an isothermal shock (see, e.g., Shu 1992).

Compressible and rotational modes. In the compressible case, the velocity field is no longer subject to the incompressibility condition, eq. (5). However, it is convenient to still decompose the field in a rotational (incompressible or solenoidal) part $\mathbf{u}_{\mathbf{k}}^{r}$ and a compressible (or potential) one $\mathbf{u}_{k}^{c}$, such that

$$
\mathbf{k} \cdot \mathbf{u}_{\mathbf{k}}^{r}=0 \quad \mathbf{k} \times \mathbf{u}_{k}^{c}=0 .
$$

These components of the velocity field can have quite distinct dynamical properties, and the energy transfer between the two is an important issue affecting the dynamics of compressible flows. Interestingly, it appears from numerical simulations of both moderately (Kida \& Orszag 1990a,b) and highly (Vázquez-Semadeni et al 1996) compressible turbulence that the transfer is predominantly from rotational to compressible modes, so that rotational energy tends to decay if no sources of vorticity $\omega \equiv \nabla \times \mathbf{u}$ are present. This is particularly interesting since turbulence is normally thought of as a rotational phenomenon.

Energy spectrum of compressible turbulence. In the highly compressible case, the presence of shocks must also be considered in the determination of the energy spectrum. A detailed derivation of the energy spectrum for a field of shocks can be found, for example, in Saffman (1971). Here we just give a brief sketch in one dimension. Consider a shock at the origin so that the 
velocity field can be described by a step function $h(x): v(x) \propto h(x)$. Then the velocity gradient is proportional to a Dirac delta function, $d v / d x \propto \delta(x)$, and its Fourier transform is then a constant, $\mathcal{F}_{k}(d v / d x)=$ cst., where $\mathcal{F}$ is the Fourier transform operator. Using the well known result for the Fourier transform of a derivative, the Fourier transform of the velocity field is then $v(k) \propto k^{-1}$, and the 1D energy spectrum is finally $E(k)=v^{2}(k) \propto k^{-2}$. Thus, the signature spectrum of a field of $1 \mathrm{D}$ shocks has a -2 slope. This result is probably related to observational properties of molecular clouds, as will be seen in $\$ 3.2$. Note that a $k^{-2}$ spectrum is also characteristic of Burgers' flows, which are solutions of the one dimensional Burgers' equation

$$
\frac{\partial u}{\partial t}+u \frac{\partial u}{\partial x}=\nu \frac{\partial^{2} u}{\partial x^{2}}
$$

which also develop ensembles of shocks. Thus, a $k^{-2}$ spectrum is also known as a Burgers spectrum (see, e.g., Saffman 1968, sect. 6).

Contrary to the incompressible case, for which the Kolmogorov spectrum is thought to be universal, in the compressible case it appears that the spectral slope may depend on the degree of compressibility of the flow. In the weakly compressible regime, 3D numerical simulations indicate that both components of the velocity develop a statistical equilibrium (Kolmogorov) spectrum (Porter et al. 1994, 1995). At intermediate compressibilities, 2D simulations indicate that the compressible component develops a $k^{-2}$ shock spectrum, while the rotational part maintains a Kraichnan equilibrium spectrum (Passot et al. 1988; Vázquez-Semadeni et al. 1995a). At very high compressibility, both components approach a $k^{-2}$ spectrum (Passot et al. 1995). The latter case is likely to be realized in the ISM, since the density contrast between the low-density intercloud medium and the densest cloud cores exceeds seven orders of magnitude.

Forcing scales and cascades in the ISM. A fundamental hypothesis in the K41 theory is that energy is injected at large scales. Since dissipation occurs predominantly at small scales, a cascade from large to small scales naturally arises. However, in the ISM, two important points are that a) energy injection occurs at small-to-intermediate scales (Scalo 1987) through supernova explosions, expanding HII regions, stellar winds and bipolar outflows, and b) in the compressible case energy can flow between compressible and rotational modes at each scale (Passot et al. 1988), and to internal energy modes as well. Furthermore, in the presence of a magnetic field, energy transfer can occur to and from magnetic modes. Thus, the concept of a cascade may not be strictly applicable to the ISM. Nonlinear transfer between different scales must still occur, but the transfer rate is not constrained to occur among solenoidal velocity modes exclusively. 
Survival of interstellar turbulence. Turbulence is a dissipative phenomenon, and a fundamental question is whether and how interstellar turbulence can be maintained. This problem is particularly important in molecular clouds, since in general CO observations exhibit highly supersonic linewidths for structures larger than $\sim 0.1 \mathrm{pc}$. Since shocks dissipate much faster than shearing motions (characteristic of incompressible turbulence), compressible turbulence in molecular clouds should decay in roughly the time it takes a strong shock to propagate across the largest scales (Goldreich \& Kwan 1974). Two principal solutions have been proposed. First, as mentioned in the previous paragraph, there are extensive stellar energy sources available for replenishing the energy. Fleck $(1980,1981)$ additionally proposed that shearing due to the Galactic differential rotation could provide the necessary energy injection. However, this possibility was not considered to be very promising (e.g., Shu et al. 1987), since it is well known that Rayleigh's criterion predicts that a rotating fluid is stable if the specific angular momentum decreases outwards (see, e.g., Shu 1992), so that no turbulence is generated from the shear. The numerical results of Vázquez-Semadeni et al. (1995) support this result. Nevertheless, it appears that energy injection from supernovae alone may be enough to maintain the turbulence at large scales in the ISM (see, e.g., the discussion by Minter \& Spangler 1997).

The second consideration is that, at the scales of molecular clouds' interiors, the presence of magnetic fields allows velocities up to the magnetosonic speed to be present without producing shocks. Since in general the magnetic field strengths are such that the Alfvén speed $v_{\mathrm{A}}$ is quite larger than the sound speed $c_{s}$ (see, e.g., Shu et al. 1987; Heiles et al. 1993), then the magnetosonic speed $v_{m}^{2}=v_{\mathrm{A}}^{2}+c_{s}^{2}$ is close to the Alfvén speed, and the latter is usually considered as the maximum speed allowed for internal motions within clouds. In many cases when magnetic field strength measurememnts are available, the inferred Alfvén speeds are indeed comparable to the velocity dispersions implied by the $\mathrm{CO}$ linewidths, suggesting equipartition between magnetic and kinetic energy (Myers \& Goodman 1988; Heiles et al 1993), and that indeed magnetic fields prevent excessive dissipation through shocks.

Note, however, that it is frequently stated (e.g., Shu et al. 1987; Mouschovias 1987) that the regime inside magnetized clouds cannot be fully turbulent, since in this case case tangled fields should be observed, while observations tend to indicate smooth field topologies. This is probably a misconception. If clouds are formed by turbulent compressions, then the field component perpendicular to the direction of compression will be amplified due to flux freezing, so that the field will tend to appear elongated in the same direction as the cloud (Passot et al. 1995). Moreover, turbulent magnetic fields with normal spectra (say $k^{-2}$, in equipartition with the velocity at all scales) have more energy at larger scales, and thus should appear smooth (i.e., dominated by the larger scales) anyway. Observations that the 
magnetic field in clouds generally blends into the smooth, larger-scale field of the complex in which the cloud is contained (Goodman et al. 1990; see also Heiles et al 1993 sect. V) are consistent with this interpretation. An alternative possibility is that the observed magnetic field orientations (using polarization measurements) do not correspond to the field within the clouds, but to an average along the entire line of sight to the cloud (e.g., Heiles et al. 1993, sect. I.C.1). In this case, no explanation in terms of large-scale power is necessary.

In summary, the above discussion on compressible turbulence suggests that it is significantly different from the incompressible case. In fact, concepts derived form the latter may sometimes not only be inadequate for the compressible case, but even misleading. In particular, the concepts of a Kolmogorov spectrum and of an energy cascade from large to small scales are probably exclusive to incompressible turbulence, even though they are often considered as the signatures of the presence of turbulence. For these reasons, it is probably appropriate to adopt a definition of turbulence based on the existence of a very large range of excited scales, with the ability to nonlinearly exchange excitation among them, as mentioned in the introduction (e.g., Lesieur 1990). We now turn to specific applications of turbulence to molecular clouds.

\section{Applications}

Turbulence is inherently a multi-scale phenomenon, and is thus related to both large-scale processes such as cloud formation and morphology, and small-scale processes such as cloud statistical properties and support against gravitational collapse. We discuss these below.

\subsection{Cloud formation}

Cloud formation in the ISM has been a long-standing problem. A variety of mechanisms have been proposed, such as coalescence of smaller clouds, various kinds of instabilities (gravitational, magnetic, thermal, etc.), and turbulence. It appears that turbulence may be responsible for the formation of the intermediate and small scale structures (molecular clouds and their clumps) while the gravitational and magnetic instabilities may be responsible for the formation of the large-scale structures (cloud complexes and superclouds) (Vázquez-Semadeni et al. 1995a,b). In this section we focus on the effects of turbulence. Detailed reviews of other processes can be found, e.g., in Elmegreen (1992, Ch. 6) and Balbus (1995) (thermal and gravitational instabilities), Zweibel (1995 - magnetic effects) and Elmegreen (1993a - coalescence models).

By definition, compressible turbulence implies the formation of density fluctuations. Gas compression (shocks, or in general, convergent flows) can 
produce either bound or transient fluctuations, and in particular an important question is whether structures formed by either turbulent compressions or passages of single shock waves can become gravitationally unstable and collpase. In this respect, Ögelman \& Maran (1976) and Elmegreen \& Lada (1977) suggested that star formation could be self-propagating, i.e., that shocks induced by supernova explosions and expanding HII regions could trigger the formation of new self-gravitating, star-forming clouds. Elmegreen \& Lada (1977) and Elmegreen \& Elmegreen (1978) discussed the gravitational instability of the compressed gas behind an isothermal shock as a physical mechanism permitting such self-propagation. Hunter \& Fleck (1982) elaborated on this by noting that the Jeans mass can be strongly reduced upon turbulent compression, considering non-isothermal cases. Vishniac (1983) refined Elmegreen \& Elmegreen's (1978) gravitational instability analysis, and also discovered a new instability, now known as the Vishniac instability, through which a shock front with thermal pressure on one side and bulk (or "ram") pressure on the other, tends to fragment. Vishniac (1994) further considered a nonlinear hydrodynamic instability in slabs bounded by shocks on both sides, showing that this instability can overwhelm the gravitational instability in the slab. This instability had already been observed in numerical simulations of shocks between colliding gas streams (Hunter et al. 1986; Stevens et al. 1992) but not properly identified. Elmegreen (1993) noted that the physical conditions of the medium before compression may determine whether compressed layers collapse or not, finding that collapse should occur in molecular clouds, but a "rebound" should occur in diffuse clouds.

As pointed out by Hunter et al. (1986), when both heating and cooling are present, the isothermal approximation, often used to describe radiative flows, is not appropriate. In cases where the heating and cooling rates are power-law functions of the density and temperature, the gas can be effectively described as a polytropic fluid $P \propto \rho^{\gamma}$, where $\gamma$ is a net polytropic exponent (e.g., Elmegreen 1992; Vázquez-Semadeni et al. 1996) and is in general different from 1 (Myers 1978). In this case, it is well known (Chandrasekhar 1961) that gravitational stability of a thermally supported spherical cloud requires $\gamma>4 / 3$. This critical value of $\gamma$ becomes 1 in 2D and 0 in 1D. McKee et al. (1993) and Vázquez-Semadeni et al. (1996) have used related arguments to consider the stability of fluid parcels compressed in $n$ dimensions by shocks or turbulence, respectively. The result is that $\gamma$ has to satisfy $\gamma<2(1-1 / n)$. Shocks are likely to have $n=1$, but generic turbulent compressions can have any dimensionality $n \leq 3$.

In summary, colliding gas streams (turbulent or otherwise) are likely to be an efficient mechanism for producing both self-gravitating and transient clouds. Note that, although self-gravitating clouds are most notoroius because they are the sites of star formation, transient clouds are also observed (e.g., Carr 1987; Falgarone \& Pérault 1988; Magnani et al. 1993) 
and may even outnumber self-gravitating ones, albeit containing less mass (Vázquez-Semadeni et al. 1997).

\subsection{Cloud structure}

A number of cloud properties are likely to be related to, or even originated by, turbulence. In this section we discuss Larson's relations, the clouds' fractal structure and their velocity distributions.

Larson's relations Larson's (1981) relations have been amply discussed in the Chapter on the Virial Theorem in this book (herafter VTC). Here, we just discuss the various types of explanations related to turbulence in molecular clouds that have been proposed for these relations.

For convenience, let us write down Larson's relations again, in their most commonly accepted form nowadays. They read

$$
\Delta v \sim R^{1 / 2} \quad \rho \sim R^{-1}
$$

where $\Delta v$ is the observed velocity dispersion in the cloud (measured by the linewidth of the observed molecular transitions, or, for densely sampled maps, by the distribution of the line centroids), $\rho$ is the cloud's average density, and $R$ is the characteristic cloud size. The linewidths become nearly thermal at scales of $\sim 0.03$ pc. Note that, as mentioned in VTC, these are definitions that originate naturally from poorly resolved observations, since the cloud often fits within the beam size, and its edges are blurred, appearing smooth and roughly circular. However, for high resolution maps, the edges of the best resolved structures are extremely amorphous, with one projected dimension in the sky probably quite disparate from the other, making it difficult to estimate a characteristic "size". Nevertheless, we assume that these quantities are meaningful in order to proceed.

As mentioned in VTC, Larson (1981) originally found slightly different exponents for the scaling relations. In particular, for the dispersion-size relation he found an exponent of 0.38 . This exponent is close to $1 / 3$, as expected for incompressible turbulence, according to the K41 theory ( $\S 2$.), leading Larson to suggest that the scaling relation is a manifestation of Kolmogorov turbulence. Subsequent determinations have pointed towards the exponents given in relations (13), although strong deviations have been found for massive and dense cores (e.g., Caselli \& Myers 1995; Plume et al 1997), as well as strongly perturbed regions (e.g., Loren 1989).

According to the discussion in $\$ 2.2$. theoretically there is no reason to expect that compressible turbulence should exhibit the same spectrum (and thus the same scaling of velocity dispersion with size) as incompressible turbulence, except in the weakly compressible case, which is not verified in molecular clouds. A number of explanations have been proposed for the $1 / 2$ exponent. One of the interpretations most directly related to turbulence 
is simply that the spectrum expected for highly compressible turbulence, $E(k) \sim k^{-2}$, appears to lead naturally to a $\Delta v \sim R^{1 / 2}$ scaling relation for the velocity dispersion. Indeed, assuming that the observed linewidths measure the mean square turbulent velocity $u_{l}$, we obtain (Passot et al 1988; Padoan 1995; Vázquez-Semadeni \& Gazol 1995; Fleck 1996; Gammie \& Ostriker 1996; Vázquez-Semadeni et al. 1997)

$$
u_{l}^{2}=2 \int_{2 \pi / l}^{\infty} E(k) d k \propto \int_{2 \pi / l}^{\infty} k^{-2} d k \propto l
$$

There are, however, a couple of caveats with this interpretation. First, the identification of $\Delta v$ with $u_{l}$ is not immediate. While the former is a direct measure of the velocity dispersion, the latter is actually the root mean square specific kinetic energy in wavenumbers larger than $2 \pi / l$, and not necessarily a physically measurable quantity. Second, $\Delta v$ is the velocity dispersion within the beam, while $u_{l}$ is an average over an idealized ensemble (and in practice, over all space; this is the so-called ergodic hypothesis). Thus, in order to decide whether the identification is correct, one should determine whether the scaling relation is observationally verified also in diffuse regions of the ISM, i.e., not only at the locations of density maxima. The data of Falgarone et al. (1992), which include positions in the sky away from brightness maxima and still exhibit a similar relation (slope 0.4), seem to support this possibility. The simulations of Vázquez-Semadeni et al. (1997) seem to also support this scenario, since the dispersion-size relation is verified even when the cloud sample includes low-density, small clouds.

On a different turbulence viewpoint, Henriksen \& Turner (1984) have proposed that an inverse cascade of angular momentum can also explain both of Larson's relations. Making the two fundamental assumptions that a) angular momentum cascades from small to large scales, so that the torque density is constant through the inertial range, and that $b$ ) the free-fall time equals the characteristic time (implying that the characteristic time is the same for all scales), they are able to derive Larson's relations. Clearly, the caveat in this approach is that the two assumptions need verification. Additionally, as pointed out in $\$ 2.2$. , compressible turbulence may globally be much less a rotational regime than incompressible turbulence (VázquezSemadeni et al. 1996), although on the other hand, vorticity is likely to be generated at small scales in the ISM, behind curved shocks (e.g., Passot \& Pouquet 1987; Fleck 1991), or within thin shock-bounded slabs (Vishniac 1994).

Explanations based on the transmission of random clump motion to the ambient medium via Alfvén waves have also been proposed. Just et al. (1994) have derived the spectrum of velocity fluctuations induced on the ambient medium by considering the differential number density of structures of a given size, from which they can derive the velocity dispersion-size scaling relation. Finally, another class of explanations are based on arguments of 
self-similarity, which we discuss in the next section, since their scope is broader than just Larson's relations.

As can be seen from the discussion above, together with the discussion in VTC, one problem concerning the dispersion-size relation is that it may be the outcome of a variety of different physical mechanisms. In fact, many other possible explanations based on mechanisms othar than the virial theorem or turbulence have not been discussed here nor in VTC. The density-size relation and the cloud mass spectrum are in the same situation. As of the present time, a unique theory of the physical processes in clouds that accounts for all their observed properties is still lacking.

Fractal structure Molecular clouds are observed to have highly complex structures, manifested in their morphology, and mass and velocity distributions. Clouds are in actuality part of a turbulent continuum with regions of higher density recursively nested within regions of lower density (hierarchical density structure) (see, e.g., the review by Scalo 1985) and cannot be considered isolated objects, except perhaps at the smallest, densest scales which decouple from their surroundings due to self-gravity. Moreover, since the medium is strongly turbulent, the clouds are naturally amorphous, and their internal velocity distributions need to be treated statistically. In fact, all of these properties of clouds appear to be fractal, i.e., exhibiting substructure at all scales down to some limit, which may or may have not been yet resolved (Goodman et al. 1997; Minter \& Spangler 1997).

\section{i) Self-similar models:}

Fractal structures are often self-similar; that is, they exhibit the same substructure properties regardless of scale. Such structures exhibit powerlaw scalings such as the Larson (1981) relations, since it can be easily shown that a power-law scale is invariant under scale transformations $x \rightarrow \lambda x$, where $x$ is the spatial coordinate and $\lambda$ is a scaling factor. Self-similar models for the ISM (e.g., Ferrini et al. (1983); Newman \& Wasserman 1990; Fleck 1996; Pfenniger 1996) have postulated a cascade of kinetic energy density (as opposed to the cascade of specific kinetic energy postulated for incompressible flows), such that its transfer rate $\epsilon_{v}$ is independent of scale, analogously to the K41 theory. Additionally, they have postulated a selfsimilarity law for the density, so that at each level $n$ of the hierarchy the density contrast with the previous level is the same. Following Fleck (1996), these two postulates read, respectively,

$$
\epsilon_{v}=\frac{\rho v^{3}}{l} \sim \text { cst. }
$$

and

$$
\frac{\rho_{n}}{\rho_{n-1}}=\left(\frac{l_{n}}{l_{n-1}}\right)^{-3 \alpha}
$$


where $\alpha$ is a "compression parameter". Note that eq. (16) becomes Larson's density-size relation for $\alpha=1 / 3$. Fleck, however, fixes $\alpha$ by obtaining from eq. (15) the form of the energy spectrum as $E(k) \sim k^{-5 / 3-2 \alpha}$ and equating it to the $k^{-2}$ spectrum for shock, also appearing in numerical simulations (Passot et al. 1995; Gammie \& Ostriker 1996), obtaining $\alpha=1 / 6$. Thus, this model predicts $\rho \sim l^{-1 / 2}$, in contrast with Larson's relation $\rho \sim l^{-1}$. Although Fleck attributes the discrepancy to the neglect of self-gravity in the model, an important additional consideration exists. As discussed in \$2.2., it is likely that in ISM turbulent cascades do not properly exist, since at each scale energy can flow out of kinetic into internal and magnetic modes. It is thus of fundamental importance to determine the mechanisms regulating such an exchange. In any case, if the ISM is a fractal, a number of the observed properties can be explained, such as the scaling relations, as explained above, the cloud mass spectrum (Elmegreen \& Falgarone 1996), and other predictions can be made; for example, ionizing radiation from massive stars could reach to much larger distances than traditionally thought (Elmegreen 1997)

Another important caveat of self-similar approaches is that it may be possible that the ISM and its structures are actually a multifractal, i.e., a fractal with different subdivision properties at each level (e.g., Chappel \& Scalo 1996).

ii) Hierarchical structure.

Concerning the fractal density structures, an extensive review has been presented by Scalo (1985). More recently, Houlahan \& Scalo (1990, 1992) have developed a numerical algorithm capable of distinguishing between randomly positioned and hierarchically nested structures, and emphasized on the limitations and distortions of traditional structure indicators like the correlation function (for the density). The method, named "structure tree analysis" by them, amounts to following the branching of the clouds into smaller subunits as higher densities (or map intensities) are considered. The method has allowed them to produce a systematic characterization of interstellar structures by properties such as the average filling factor of the dense regions, and the mass efficiency of fragmentation per level of the hierarchy.

A number of mechanisms have been proposed as originators of the hierarchical structure in the ISM (again, see Scalo 1985 for a review). In the framework of turbulence, it has been normally postulated that, being a selfsimilar phenomenon (as indicated by the power-law scalings of the energy spectrum and the velocity differences - see \$2.1.), it should produce selfsimilar density structures as well, as in the models of Ferrini et al. (1983) and Fleck (1996). A first step towards a mechanism arising from turbulence was suggested by Vázquez-Semadeni (1994), in which the turbulence is assumed to produce density fluctuations with a specific (but unknown) probability distribution function (pdf). He suggested that hierarchical structure should 
arise naturally provided the pdf $f$ satisfies a) $f$ does not depend on the local average density, but only on the fractional density increment $\delta=d \rho / \rho$, and b) $f\left(\delta^{n}\right)<f^{n}(\delta)$, where $n$ is the number of density increment steps to reach a final density. That is, if the probability of reaching a given large density increment $\Delta$ is larger when it is reached by a succession of small steps $\delta$ than when it is reached through a single density jumps, then one should expect the highest densities to occur within regions of previously enhanced density. Of course, in this approach, the physical mechanism determining the specific form of the pdf is still lacking.

iii) Fractal cloud boundaries.

Yet another apparent manifestation of turbulence in the shaping of clouds is given by their extremely complicated morphologies. A well known feature of turbulent flows is that they produce much more efficient mixing than that occurring in laminar flows. In the incompressible case, such mixing occurs via the stretching of fluid parcels which intertwine with other parcels due to shear. Even though in the highly compressible case we have mentioned the global subdominance of shearing (solenoidal) motions, in shocks significant vorticity production is expected (Hayes 1957; Passot \& Pouquet 1987; Fleck 1991; Vishniac 1994), so we should expect complicated boundaries in clouds formed by turbulent shock compressions.

Scalo (1990) and Falgarone et al. (1991) have measured the fractal dimension of a large sample of clouds by measuring their projected areas and perimeters in the sky. In a simple geometrical object, such as a circle or a square, the perimeter $P$ is proportional to the square root of the area $A$. However, fractal objects (e.g., Mandelbrot 1977) that exhibit substructure at each resolution have perimeters that increase faster with the area. Indeed, Falgarone et al. find that $P \propto A^{0.68}$, indicating a significant fractality. The only caveat with this result is that they mention that the slope of 0.68 is quite similar to that found for clouds in the Earth's atmosphere, which are subject to very different physical processes. Thus, it may be that the fractal dimension is not enough to distinguish between various kinds of flow regimes.

iv) Velocity distributions and intermittency.

Clearly, the velocity field is a major protagonist in a turbulent description of the ISM and molecular clouds. Yet, in the velocity dispersion-size relationship of Larson's (1981) relations, only the linewidth of the transition is generally involved, rather than actual velocity measurements. In fact, linewidths refer to only one observation per cloud or clump. Several workers have attempted to improve on this by measuring the actual radial velocities, obtained from line centroids in densely sampled maps. Such measurments allow determining statistical indicators such as the correlation function and correlation length, providing a characterization of the actual velocity fields in clouds. Scalo (1984) emphasized these points and attempted measur- 
ing the velocity correlation and structure functions, although he found only very weak correlations, suggesting a very small correlation length, $\leq 0.05 \mathrm{pc}$. Kleiner \& Dickman (1984, 1985) and Dickman \& Kleiner (1985) presented the theoretical formalism, and performed a detailed study of the Taurus molecular complex, finding a characteristic length scale for the density, but still no correlation length for the velocity with a resolution of $0.6 \mathrm{pc}$. More recently, Miesch \& Bally (1994) have been able to measure correlation lengths, albeit noting that the latter are strongly influenced by the range of scales sampled by the observations, and conclude that this is a manifestation of the self-similarity of the flow.

An important phenomenon known to occur in incompressible turbulence is intermittency, consisting of strong, sporadic, unpredictable fluctuations in the velocity (see, e.g., Lesieur 1990; Frisch 1995). This phenomenon manifests itself in non-Gaussian distributions of the velocity differences and derivatives. Searches for this behavior have been conducted by Falgarone \& Phillips (1990) and Miesch and Scalo (1995) for large samples of clouds. The former authors used line profiles as indicators, while the latter used again pdfs of the line centroids. Interestingly, these groups have found nonGaussian velocity statistics, with exponential distribution tails. This results contrasts with those found in numerical simulations of compressible turbulence (Passot et al. 1995; Lis et al. 1996), for which the velocities exhibit nearly Gaussian distributions, while the derivatives exhibit non-Gaussian profiles (Miesch \& Scalo 1995; Lis et al. 1996). Thus, the origin of exponential wings in interstellar velocity distributions is an open problem. Miesch and Scalo (1995) discuss a number of possible mechanisms, but without being able to decide among them.

Cloud support. As mentioned in VTC, turbulence at the small scales $m i$ croturbulence) can provide support against gravitational collapse, in addition to that provided by thermal pressure and the magnetic field. In fact, as mentioned before, the linewidths are generally supersonic, so thermal support is negligible at all but the smallest scales. Since magnetic support has been discussed at length in VTC, we restrict ourselves here to a brief review of the turbulent support exclusively.

Turbulent pressure was first considered as an agent of cloud support by Chandrasekhar (1951), who considered a total pressure of the form $P=$ $\rho\left(c^{2}+\sigma^{2} / 3\right)$ in the classical Jeans (1902) gravitational instability analysis. Here $c$ is the sound speed and $\sigma$ is the turbulent velocity dispersion. This procedure simply enlarges the Jeans length by a small factor, but gives no qualitatively new results. Bonazzola et al. (1987) recognized that, since the turbulent energy spectrum indicates that larger eddies contain larger turbulent energies, the scale dependence should be introduced in the Jeans analysis. Indeed, they found that for sufficiently steep spectrum slopes $\left(E(k) \propto k^{-n}\right.$, with $n \geq 3$ ), the Jeans criterion is reversed, so that small scales are unstable 
and large scales are stable. This in fact is qualitatively correct, since star formation in the ISM occurs in the smallest cores. However, their required spectral slopes are too steep, since 3D compressible turbulence is expected to have slopes $5 / 3 \leq n \leq 2$ (see \$2.2.). Just et al. (1994) and Vázquez-Semadeni \& Gazol (1995) have noted that the Bonazzola et al. analysis neglects the clumpiness of the ISM, and introduced a power-law dependence for the density scales. This analysis essentially recovers the virial equilibrium condition. Just et al., using a particular model of excitation of MHD waves from clump motions within a cloud, obtain a $\Delta v \sim R^{1 / 2}$ relation, and thus recover the standard exponents for Larson's relations. However, Vázquez-Semadeni \& Gazol take the turbulent spectral index $\alpha$ as a free parameter, and emphasize that any combination such that $\alpha+\beta=3$, where $\beta$ is the exponent in the density-size scaling relation, suffices to achieve virial equilibrium. Thus, Larson's relations are just one of an infinite number of possible virial equilibrium configurations. However, the latter authors also emphasize that their analysis clearly does not apply to all regions in the ISM, but only to density peaks that follow the specified scaling law for the density. Lower-density regions away from the peaks necessarily do not follow this scaling relation. A similar study has been performed by Vranjes (1994), although assuming linear, scale-independent density fluctuations.

Turbulent cloud support was tested numerically by Léorat et al. (1990), who performed simulations of forced self-gravitating compressible turbulence and looked for the conditions necessary for support to be effective. They found that gravitational collapse may be prevented if the turbulent forcing was injected at sufficiently small scales. This contrasts with the results of Vázquez-Semadeni et al. (1996), in which gravitational collapse was induced by turbulent forcing at large scales. Thus, turbulence has a twofold character: small turbulent scales can help prevent gravitational collapse, while large scales can promote it. However, the large scale components of the turbulence are often neglected when considering this problem, and turbulence is regarded as just providing support, without the ability of distorting the cloud morphology and mass distribution.

\subsection{General interstellar turbulence theory}

In this section we wish to describe very briefly some other existing theoretical work, highlighting their results, as well as their limitations and, in some cases, flaws. Unfortunately, we have to make the disclaimer here that this section is by no means complete, but only presents a random collection of work which we consider of interest.

McKee \& Zweibel (1992) and Zweibel \& McKee (1995) have studied the energy budget of clouds, first by producing an Eulerian form of the Virial Theorem suitable for analysis of turbulent clouds, and then providing support for the possibility that clouds should exhibit energy equipartition. In particular they consider the possibility of turbulent pressure confinement. 
However, their results in this respect are questionable, since they consider confinement by small scale turbulent modes only, while assuming that large scale modes will only have the effect of sending shocks through the cloud, thus neglecting the possibility that the large scale modes actually have the ability of distorting or disrupting the cloud. Thus, their analysis is restricted only to the densest cores which have decoupled from their surroundings due to self-gravity.

Sridhar \& Goldreich (1994) and Goldreich \& Sridhar (1995) have attempted to produce a theory of interstellar turbulence based on the assumption that it consists of nonlinear interactions among shear Alfvén waves. One of the influencing results of this theory is that the turbulent "eddies" should be elongated in the direction of the magnetic field at small scales. Such elongations have in fact apparently been detected (Desal et al. 1994). However, the theory has two major problems. One, it assumes incompressibility, so it is at most applicable to the diffuse ISM. The same applies to a recent attempt by Norman \& Ferrara (1997) to characterize a universal forcing function for interstellar turbulence considering the various types of energy sources. Two, it has been claimed by Matthaeus \& Montgomery (1995) that the theory contains a fundamental error concerning the nature of the nonlinear interactions.

Padoan (1995) has produced a phenomenological theory that predicts the protostar formation efficiency and mass distribution as functions of the parameters of the turbulent ambient medium, such as its average density, temperature, rms turbulent velocity and postshock cooling time. However, the theory is phenomenological in that it assumes specific forms for the gas density pdf and the mass spectrum of the clumps, guided by the results of numerical simulations, rather than deriving them.

Finally, it is perhaps appropriate to conclude this section noting that De Vega et al. (1996) have pointed out that self-similar structure could arise in the ISM by purely gravitational effects, without the need for turbulence.

\section{Conclusions}

In this course we have reviewed the basic theory of incompressible and compressible turbulence, and then attempted to describe the wide variety of interstellar and molecular cloud phenomena which are likely to be related to turbulence, in particular the processes of cloud formation, cloud structuring and cloud support. It should be emphasized that most treatments are phenomenological, since a full theory of interstellar turbulence is lacking. Such a theory should be able to predict properties like the filling factor of the dense regions, the density pdf, the density power spectrum and correlation function, and the density contrasts at each hierarchical level (rather than assuming them); the velocity spectrum and correlation as a function of the 
available energy sources, and the efficiency of star formation. However, since an equivalent theory is not available even in the incompressible case, it is clear that interstellar turbulence research is still in a highly incipient phase.

Acknowledgments. The author is glad to acknowledge J. Scalo and T. Passot for helpful comments and a critical reading of the manuscript.

\section{References}

Armi, L. \& Flament, P. 1985, J. Geophys. Res., 90, no. C6, 11779

Balbus, S. A. 1995, in Physics of the Interstellar Medium and Intergalactic Medium, ed. A. Ferrara, C. F. McKee, C. Heiles and P. Shapiro (San Francisco: A.S.P), 328

Batchelor, G. K. 1953, The Theory of Homogeneous Turbulence (Cambridge: Univ. Press)

Bonazzola, S., Falgarone, E., Heyvaerts, J., Pérault, M., Puget, J. L. 1987, A\&A 172, 293

Brachet, M. E., Meneguzzi, M., Politano,H. \& Sulem, P. L. 1988, J. Fluid Mech. 194, 333

Carr, J. S. 1987, ApJ, 323, 170

Caselli, P. \& Myers, P. C. 1995, ApJ, 446, 665

Chandrasekhar, S. 1951, Proc. R. Soc. London, 210, 26

Chandrasekhar, S. 1961, Hydrodynamic and Hydromagnetic Stability (Oxford: Clarendon Press)

Chappel, D. \& Scalo, J. 1996, preprint

Cowling, T. G. 1976, Magnetohydrodynamics (Bristol: Adam Hilger Ltd.)

Currie, I. G., 1974, Fundamental Mechanics of Fluids (New York: McGrawHill)

Desal, K. M., Gwinn, C. R., \& Diamond, P. J. 1994, Nature, 372, 754

De Vega, H. J., Sánchez, N., \& Combes, F. 1996, Nature, 383, 56

Dickman, R. L. 1985, in Protostars and Planets II, ed. D. C. Black \& M. S. Matthews (Tucson: Univ. of Arizona Press), 150

Elmegreen, B. G., 1992, in The Galactic Interstellar Medium. SAAS-FEE Advanced Course 21, ed. D. Pfenniger and P. Bartholdi (Berlin: Springer-Verlag), 157

Elmegreen, B. G. 1993a, in Protostars and Planets III, ed. E. H. Levy and J. I. Lunine (Tucson: The University of Arizona Press), 97

Elmegreen, B. G. 1993b, ApJ, 419, L29

Elmegreen, B. G. \& Lada, C. J. 1977, 1977, ApJ, 214, 725

Elmegreen, B. G. \& Falgarone, E. 1996, ApJ, 471, 816 
Elmegreen, B. G. 1997, ApJ, in press

Falgarone, E. \& Puget, J. L. 1986, A\& A, 162, 235

Falgarone, E. \& Pérault, M. 1988, A\& A, 205, L1

Falgarone, E. \& Phillips, T. 1990, ApJ, 359, 344

Falgarone, E., Phillips, T. G., \& Walker, C. K. 1991, ApJ, 378, 186

Falgarone, E., Puget, J.-L., \& Pérault, M. 1992, A\& A, 257, 715

Fleck, R. C. 1980, ApJ 242, 1019

Fleck, R. C. 1981, ApJ 246, L151

Fleck, R. C. 1991, Ap\&SS, 182, 81

Fleck, R. C. 1996, ApJ, 458, 739

Frisch, U. 1995, Turbulence. The Legacy of A. N. Kolmogorov (Cambridge: University Press)

Frisch, U. \& Sulem, P. L. 1984, Phys. Fluids, 27, 1921

Gammie, C. \& Ostriker, E. 1996, ApJ, 466, 814

Goldreich, P. \& Kwan, J. 1974, ApJ, 189, 441

Goodman, A. A., Bastien, P., Myers, P. C. \& Menard, F. 1990, ApJ, 359, 363

Goodman, A. A., Barranco, J. A., Wilner, D. J. \& Heyer, M. H. 1997, ApJ, submitted

Grant, H. L., Stewart, R. W. \& Moilliet, A. 1962, J. Fluid Mech. 12, 241

Hayes, W. D. 1957, J. Fluid Mech., 2, 595

Heiles, C., Goodman, A., McKee, C. F. \& Zweibel, E. G. 1993, in Protostars and Planets III, ed. E. H. Levy and J. I. Lunine (Tucson: The University of Arizona Press), 279

Henriksen, R. N. \& Turner, B. E. 1984, ApJ, 287, 200

Herring, J. R., Orszag, S. A., Kraichnan, R. H. \& Fox, D. G. 1974, J. Fluid Mech., 66, 417

Houlahan, P. \& Scalo, J. 1990, ApJS, 72, 133

Houlahan, P. \& Scalo, J. 1992, ApJ, 393, 172

Hunter, J. H. Jr., \& Fleck, R. C. 1982, ApJ, 256, 505

Hunter, J. H. Jr., Sandford, M. T. II, Whitaker, R. W. \& Klein, R. I.1986, ApJ, 305, 309

Jeans, J. H. 1902, Phil. Trans. R. Soc. London, A 199, 1

Just, A., Jacobi, S., \& Deiss, B. M. 1994, A\& A, 289, 237

Kida S., Orszag S.A., 1990a, J. of Sci. Comp., 5, 85

Kida S., Orszag S.A., 1990b, J. of Sci. Comp., 5, 1

Kolmogorov, A. N. 1941, Dokl. Akad. Nauk., 30, 301

Kraichnan, R. H. 1967, Phys. Fluids 10, 1417 
Kraichnan, R. H. \& Montgomery, D. 1980, Rep. Prog. Phys., 43, 547

Lesieur, M. 1990, Turbulence in Fluids, $2^{\text {nd }}$ ed. (Dordrecht: Kluwer)

Lis, D. C., Pety, J., Phillips, T. G. \& Falgarone, E. 1996, ApJ 463, 623

Magnani, L., LaRosa, T. N., \& Shore, S. N. 1993, ApJ, 402, 226

Malerud, S., Maloy, K. J.,\& Goldburg, W.I. 1995, Phys. Fluids 7, 1949

Mandelbrot, B. B. 1977, Fractals (San Francisco: Freeman)

McKee, C. F., Zweibel, E. G., Goodman, A. A., \& Heiles, C., in Protostars and Planets III, ed. E. H. Levy \& J. I. Lunine (Tucson: Univ. of Arizona Press), 327

Miesch, M. S. \& Bally, J. 1994, ApJ, 429, 645

Miesch, M. S. \& Scalo, J. 1995, ApJ, 450, L27

Minter \& Spangler 1977, ApJ submitted

Mouschovias, T. Ch. 1987, in Physical Processes in Interstellar Clouds, ed. G. E. Morfill and M. Scholer (Dordrecht: Reidel), 453

Myers, P. C. 1978, ApJ, 225, 380

Myers, P. C., \& Goodman, A. A. 1988, ApJ, 326, L27

Newman, W. I., \& Wasserman, I. 1990, ApJ, 354, 411

Norman, C. \& Ferrara, A. 1997, preprint

Obukhov, A. M. 1941, C. R. Acad. Sci. USSR, 32, 19

Ögelman, H. B. \& Maran, S. P. 1976, ApJ, 209, 124

Ott, E., 1993, Chaos in Dynamical Systems (Cambridge: University Press)

Padoan, P. 1995, MNRAS, 277, 377

Passot, T., \& Pouquet, A. 1987, J. Fluid Mech., 181, 441

Passot, T., Pouquet, A., \& Woodward, P. R. 1988, A\&A, 197, 228

Passot T., Vázquez-Semadeni E., Pouquet A., 1995, ApJ, 455, 702

Pfenniger, D. 1996, preprint

Porter, D. H., Pouquet, A., Woodward, P. R. 1994, Phys. Fluids, 6, 2133

Porter, D. H., Pouquet, A., Woodward, P. R. 1995, in Small-Scale Structures in Three-Dimensional Hydrodynamic and Magnetohydrodynamic Turbulence, ed. M. Meneguzzi, A. Pouquet, P.-L. Sulem (Berlin: Springer), 51

Rose, H. A. \& Sulem, P. L. 1978, J. Physique, 39, 441

Saffman, P. G. 1968, in Topics in Nonlinear Physics, ed. N. J. Zabusky (New York: Springer-Verlag), 485

Saffman, P. G. 1971, Stu. Appl. Math., 50, 377

Scalo, J. 1984, ApJ, 277, 556

Scalo, J. 1985, in Protostars and Planets II, ed. D. C. Black and M. S. Matthews (Tucson: Univ. of Arizona Press), 201 
Scalo, J. M. 1987, in Interstellar Processes, ed. D. J. Hollenbach and H. A. Thronson (Dordrecht: Reidel), 349

Scalo, J. 1990, in Physical Processes in Fragmentation and Star Formation, ed. R. Capuzzo-Dolcetta, C. Chiosi, \& A. di Fazio (Dordrecht: Kluwer), 151

Shore, S. N. 1992, An Introduction to Astrophysical Hydrodynamics (San Diego: Academic Press)

Shu, F. 1992, Gas Dynamics (Mill Valley: University Science Books)

Shu, F., Adams, F. C., Lizano, S. 1987, ARAA, 25, 23

Spangler, S. R. 1991, ApJ, 376, 540

Spitzer, L., Jr., 1978, Physical Processes in the Interstellar Medium (New York: Wiley-Interscience)

Stevens, I. R., Blondin, J. M \& Pollock, A. M. T. 1992, ApJ 386, 265

Tennekes, H. \& Lumley, J. L. 1972, A First Course in Turbulence (Cambridge: MIT Press)

Vázquez-Semadeni, E. 1994, ApJ, 423, 681

Vázquez-Semadeni, E., Passot T., Pouquet A., 1995a, ApJ, 441, 536

Vázquez-Semadeni, E., Passot T., Pouquet A., 1995, in Proceedings of the Fifth Mexico-Texas Conference on Astrophysics: Gaseous Nebulae and Star Formation, Rev. Mex. A.A. Ser. Conf., 3, 61.

Vázquez-Semadeni, E., Passot T., Pouquet A., 1996, ApJ in press (Dec. 20)

Vázquez-Semadeni, E., Ballesteros-Paredes, J. \& Rodríguez, L. F. 1997, ApJ, in press (Jan. 1)

Vranjes, J. 1994, Ap\& SS, 213, 139

Zweibel, E. 1995, in Physics of the Interstellar Medium and Intergalactic Medium, ed. A. Ferrara, C. F. McKee, C. Heiles and P. Shapiro (San Francisco: A.S.P), 524 